\title{
Concentrations of four fecal steroids in wild baboons: short-term storage conditions and consequences for data interpretation
}

\author{
J.W. Lynch, ${ }^{\mathrm{a}, *}$ M.Z. Khan, ${ }^{\mathrm{b}}$ J. Altmann, ${ }^{\mathrm{a}, \mathrm{c}, \mathrm{d}}$ M.N. Njahira, ${ }^{\mathrm{e}}$ and N. Rubenstein ${ }^{\mathrm{a}}$ \\ ${ }^{a}$ Department of Ecology and Evolutionary Biology, Princeton University, Princeton, NJ 08544, USA \\ ${ }^{\mathrm{b}}$ Department of Biology and Microbiology, University of Wisconsin-Oshkosh, Oshkosh, WI 54901, USA \\ ${ }^{\mathrm{c}}$ Institute of Primate Research, National Museums of Kenya, Nairobi, Kenya \\ d Department of Conservation Biology, Chicago Zoological Society, Brookfield, IL, USA \\ ${ }^{\mathrm{e}}$ National Museums of Kenya, Nairobi, Kenya
}

\begin{abstract}
One source of both bias and "noise" in fecal steroid analysis is temporal change in steroid concentrations resulting from duration or conditions of fecal sample storage. However, no consensus currently exists regarding correct procedures or precautions necessary for fecal sample storage, and conditions vary widely within field endocrinology literature. This study considered the effects of shortterm, weeks-long, storage conditions on quantifiable fecal testosterone (fT), glucocorticoids (fGC), estrogens (fE), and progestagen (fP) metabolite concentrations in wild baboons (Papio cynocephalus). Quadruplicate subsamples of fecal samples $(n=29)$ collected at Amboseli National Park and its environs were subjected to four different storage conditions prior to lyophilization, in order to determine the effects of storage on subsequent steroid concentrations, as assessed by ${ }^{125}$ I radioimmunoassays. As expected, the best alternative to the "initial condition" of lyophilization at three days after collection was to freeze fecal samples at $-20^{\circ} \mathrm{C}$ for two weeks prior to lyophilization. This storage method resulted in no significant change from initial steroid concentrations for fE, fT, or $\mathrm{fP}$, although fGC showed a slight but significant decline. Storage for two weeks in a charcoal refrigerator caused a mean increase in all four steroid concentrations. However, the results from this storage condition were robust in terms of practical questions asked of the data: fE and fP values still reflected pregnant versus non-pregnant states in baboon females; a fGC profile constructed by age class resembled that created from the samples from the initial condition, although slightly inflated across age classes; and there were only moderate changes in relative fT concentrations across adult males. Knowledge of the effects of storage upon each steroid analyzed within one's study is a necessary component in determining the optimal compromise for storage protocol in a particular research project.
\end{abstract}

(c) 2003 Elsevier Science (USA). All rights reserved.

Keywords: Fecal steroids; Field storage; Glucocorticoids; Progesterone; Testosterone; Estrogen; Baboons; Papio

\section{Introduction}

The goal of initial field applications of fecal steroid analysis was the detection of relatively gross changes in hormonal concentrations, such as ovarian cycling patterns or the changes in reproductive hormones from cycling to pregnancy (for reviews, see Lasley and Kirkpatrick, 1991; Schwarzenberger et al., 1997; Whitten et al., 1998). These initial applications continue for an

\footnotetext{
${ }^{*}$ Corresponding author. Fax: 1-609-258-2712.

E-mail address: jwlynch@ucdavis.edu (J.W. Lynch).
}

increasing number of mammalian taxa (e.g., carnivores: Wasser et al., 1995; proboscids: Wasser et al., 1996; primates: Campbell et al., 2001; Heistermann et al., 2001; Jurke et al., 1997; Strier and Ziegler, 1994; Ziegler et al., 2000; ungulates: Garnier et al., 1998; Möstl et al., 1999), and as fecal steroid analysis has become a more widely appreciated field technique, applications have expanded to consider steroid concentrations in relation to reproductive seasonality (Brockman et al., 2001; Cavigelli, 1999; Lynch et al., 2002; Strier et al., 1999; Ziegler et al., 2000), stress (Boinski et al., 1999; Creel et al., 1996; Dehnhard et al., 2001; Goymann et al., 1999), ontogeny (Strier and Ziegler, 2000), rank (Kraus 
et al., 1999), and mating and aggression in males (Brockman et al., 1998; Lynch et al., 2002; Ostner et al., 2002). In these contexts, glucocorticoids and androgens have joined female reproductive hormones as markers of interest for fecal steroid analysis.

As a non-invasive method for repeated individual sampling, fecal steroid analysis has enormous potential for answering both longitudinal and populational questions about the endocrinology of wild animals. In many cases, however, these types of investigations require the detection of subtle differences in hormone concentrations among individuals and through time. When gross patterns are examined, fecal steroid profiles often appear "noisy," with high variation around mean values, in comparison to urine or serum profiles (Boinski et al., 1999; Heistermann et al., 1996; Shideler et al., 1994; Ziegler et al., 1996). Therefore, when more subtle patterns are the target of interest, minimizing this noise is essential in order to detect real biological differences with strong resolution. One potential source of "noise" is the change in fecal steroid concentration as a result of the conditions of sample storage (Washburn and Millspaugh, 2002; Whitten et al., 1998). A related issue is that of directional change in steroid concentration through time in storage, which could lead to bias in sample values based on storage duration (Khan et al., 2002). In this case the risk is that variation in steroid concentrations might be misinterpreted as a biological rather than methodological phenomenon.

At present, there is no consensus about the correct procedures or precautions necessary for fecal sample storage, and the conditions and duration of sample storage vary widely within field endocrinology literature (reviewed in Khan et al., 2002). Experiments on the effects of medium-term storage on fecal steroids (e.g., Khan et al., 2002; Terio et al., 2002; Washburn and Millspaugh, 2002) have lagged behind those for urinary steroids (e.g., Brown et al., 1995; Grant and Beastall, 1983; Kesner et al., 1995), despite the recognition that, due to the bacterial load in fecal matter, steroids are inherently less stable in feces than in urine (Whitten et al., 1998), and in fact, steroid levels can change within hours following defecation if samples are not immediately preserved (Möstl et al., 1999). This study considers the effects of short-term, weeks-long, storage conditions on quantifiable fecal testosterone (fT), glucocorticoid (fGC), estrogen (fE), and progestagen (fP) concentrations in wild baboons (Papio cynocephalus).

Ethanol has been validated as an hours-long preservative of steroids in baboon feces, both at sub-zero and at ambient temperatures (Wasser et al., 1988), and fecal steroids have been considered most stable over time when stored in 95\% ethanol at sub-zero temperatures (Whitten et al., 1998). However, some fecal steroid metabolites exhibit changes in concentration after months of storage, even when kept at $-20^{\circ} \mathrm{C}$ (Khan et al., 2002). Baboon fecal samples stored in ethanol over a six-month period changed significantly in $\mathrm{fE}$ and fGC concentrations, with both steroids approximately doubling in value at the apex of the steroid increase at ambient temperature, and showing a more dampened but significant increase and subsequent decline when kept in a $-20^{\circ} \mathrm{C}$ freezer (Khan et al., 2002). These data suggest that when prompt extraction or lyophilization of fecal steroids is not possible, the optimal conditions for samples would be short-term sub-zero storage in ethanol, followed by lyophilization (Khan et al., 2002). Nevertheless, practical constraints of field research may necessitate at least some storage at less than optimal conditions prior to processing.

The present experiment considers the effects of different short-term storage conditions prior to lyophilization and extends the findings of prior work in our lab in three important ways. First, our findings that fGC and $\mathrm{fE}$ concentrations exhibited different responses to a given storage regime indicated that each hormone of interest must be verified for storage effects (Khan et al., 2002). Here we continue validation of storage techniques for $\mathrm{fE}$ and $\mathrm{fGC}$, as well as two additional hormones of interest, fT and fP. Second, significant differences in fecal steroid metabolite concentrations were observed when samples were stored for 30 days, at either ambient laboratory temperature (fE) or at sub-zero temperatures (fGC; Khan et al., 2002). Therefore here we examine changes in fecal steroid metabolite concentrations within the first 15-30 days of storage in order to determine the optimal short-term storage regime for samples analyzed for multiple hormones. Third, the present study uses samples that differ in initial concentrations and that were collected under field conditions, in order to assess the effect of storage condition on fecal steroid concentrations in a broad sampling from a wild population.

\section{Materials and methods}

\subsection{Experimental conditions for fecal storage}

Twenty-nine fecal samples were collected from a cross-section of known individuals in the Amboseli baboon population, including 14 males and 12 females that ranged in age from 5 to 16 years. These samples were collected on December 1, 2001, and December 15, 2001. After a fecal sample had been very well mixed in a small cup with a wooden stirrer, subsamples were placed into four identical vials pre-filled with $95 \%$ ethanol, to obtain an approximately 2:5 volumetric ratio of feces to ethanol. Each of the quadruplicate vials was labeled with the individual's identification code and date of collection, and was randomly assigned to condition "I," "A," "B" 
or "C." The "I" ("Initial") sample was transported to Nairobi two days after collection and lyophilized at the National Museums of Kenya (NMK). In all cases, prior to lyophilization, samples were placed in a fume hood and the ethanol was evaporated over a period of 1-3 days. Sample "A" was transported to NMK along with sample "I," and was frozen two days after collection. Sample "A" remained in the $-20^{\circ} \mathrm{C}$ freezer in Nairobi for two weeks prior to ethanol evaporation and lyophilization. Samples "B" and "C" were both stored for those two weeks in the $95 \%$ ethanol solution in Amboseli's charcoal refrigerator, which maintains an average daily temperature of about $20^{\circ} \mathrm{C}$ and a range from 15 to $25^{\circ} \mathrm{C}$, a more stable storage condition than ambient in an tropical savannah environment where maximum temperature can sometimes exceed $40^{\circ} \mathrm{C}$. After a total of 16 days in the charcoal refrigerator, these samples were transported to NMK, at which time sample "B" was immediately processed for lyophilization and sample "C" was stored in the $-20^{\circ} \mathrm{C}$ freezer for two more weeks prior to the lyophilization process (see Table 1). Subsequently, all freeze-dried quadruplicate samples were stored at $-20^{\circ} \mathrm{C}$ in Nairobi until transport to the United States upon completion of the protocol. All samples (from all conditions, $n=116$ ) were handcarried together to Princeton University, for subsequent analysis.

\subsection{Radioimmunoassay}

After transport to Princeton University, each fecal sample was sifted to remove vegetative matter and $0.2 \mathrm{~g}$ fecal powder was extracted into $2 \mathrm{ml} 90 \%$ methanol, then run through an Oasis cartridge for solid phase extraction, in preparation for assay (Khan et al., 2002). All samples were methanol extracted within two weeks of arrival in Princeton, and all replicates (I, A, B, and C) from a given sample were extracted together on the same day.

Concentrations of four classes of steroids, glucocorticoids, estrogens, progestagens, and androgens, were assessed in the baboon fecal samples, each through ${ }^{125} \mathrm{I}$ radioimmunoassays. All samples from each condition were assayed in duplicate, and mean concentrations expressed as nanograms of hormone per gram of dry fecal matter (Wasser et al., 1993). All quadruplicates (I, $\mathrm{A}, \mathrm{B}$, and $\mathrm{C}$ ) for a particular sample were run within the same assay for each steroid.
Extraction efficiencies. Methanol extraction recoveries were assessed by adding $10,000 \mathrm{cpm}{ }^{125}$ I-labeled hormone to dry feces and incubating the mixture at ambient temperature for $1 \mathrm{~h}$ prior to methanol extraction. Methanol extraction recoveries were $85.0 \pm 0.5 \%(n=$ 10) for corticosterone, $92.3 \pm 0.5 \%(n=10)$ for estradiol, $86.9 \pm 0.7 \%(n=10)$ for progesterone, and $91.9 \pm 0.8 \%$ $(n=10)$ for testosterone. Solid phase extraction recovery for each hormone was assessed by assaying duplicate samples before and after solid phase extraction, yielding a recovery of $89.1 \pm 1.3 \%(n=10)$ for estradiol, $86.9 \pm$ $3.0 \%(n=8)$ for corticosterone, $102.7 \pm 5.1 \%(n=10)$ for progesterone, and $76.6 \pm 4.4 \%(n=5)$ for testosterone.

Fecal glucocorticoids. Radioimmunoassays were run using the ${ }^{125} \mathrm{I}$ kit of Corticosterone for Rats and Mice (ICN Diagnostics, Costa Mesa, CA) modified with methods described in Khan et al. (2002). Each standard curve tube was coated with $5 \mu$ doubly charcoal stripped fecal pool, in order to improve assay accuracy. Intraand interassay coefficients of variation for the corticosterone assays $(n=3)$ were 3.1 and $14.3 \%$ for a control standard, and 1.2 and $10.9 \%$ for a mixed male-female fecal pool.

Fecal estrogens. Estrogen assays were run using an ${ }^{125}$ I Total Estrogens Kit (ICN Diagnostics, Costa Mesa, CA) following methods described in Khan et al. (2002). Intra- and interassay coefficients of variation for the estrogen assays $(n=3)$ were 3.3 and $10.4 \%$ for a fecal pool.

Fecal progestagens. Progestagens were analyzed using an ${ }^{125}$ I Direct Progesterone Kit (Pantex, Santa Monica, CA). Cross- reactivity for the primary antibody is $100 \%$ for progesterone, $0.5 \%$ for $17 \alpha$-hydroxyprogesterone, and $0.1 \%$ for androstenedione. Standard concentrations ranged from $20 \mathrm{pg}$ to $8 \mathrm{ng}$ per tube. A $20 \mu 1$ aliquot of each solid-phase extracted fecal sample was dried with air in a water bath and resuspended in $100 \mu \mathrm{l} \mathrm{PBS} /$ bovine serum albumin buffer. Samples and standards were incubated with $100 \mu$ of tracer and $100 \mu 1$ of primary antibody (diluted 1:6) overnight. On the following day $100 \mu \mathrm{l}$ of secondary antibody was added, and the tubes were vortexed and incubated for $1 \mathrm{~h}$ at room temperature. After $1 \mathrm{~h}$ of centrifugation at $2 g$, the supernatant was aspirated and the pellet was counted in a gamma counter. A serial dilution of a solid-phase extracted baboon fecal sample showed parallelism to the progesterone standard curve,

Table 1

Experimental conditions for fecal sample storage

\begin{tabular}{|c|c|c|c|c|}
\hline & I (initial) & A & $\mathrm{B}$ & $\mathrm{C}$ \\
\hline Charcoal refrigerator & 2 days & 2 days & 16 days & 16 days \\
\hline Frozen $\left(-20^{\circ} \mathrm{C}\right)$ & 0 days & 14 days & 0 days & 14 days \\
\hline Lyophilized & After 2 days & After 16 days & After 16 days & After 30 days \\
\hline
\end{tabular}


spiked with $5 \mu \mathrm{l}$ doubly charcoal stripped fecal extract $(t=0.68, p=0.50)$. Accuracy for added baboon fecal extract to the standard curve points was a mean of $109.2 \% \pm \mathrm{SE} 3.6(n=7)$. For $\mathrm{fP}$ assays $(n=6)$, the intra- and interassay coefficients of variation were 5.0 and $7.9 \%$ for a low male-female fecal pool and 2.3 and $11.2 \%$ for a high male-female fecal pool.

Fecal testosterone. Our testosterone assay used reagents from the ${ }^{125}$ I Equate Testosterone RIA Kit (SolidPhase, South Portland, ME). Cross-reactivity of the Equate testosterone antibody was tested by subjecting a pooled male baboon fecal extract to HPLC and then assaying collected fractions with the RIA Kit (performed by P. Whitten, Emory University). The fractions containing the testosterone peak were responsible for over $99 \%$ of the immunoreactivity.

The slope of a serial dilution (1:2 to 1:32) of a male fecal extract pool paralleled that of the standard curve $(t=1.12, p=0.27)$. Accuracy for added baboon fecal extract to the standard curve points was a mean of $105.4 \% \pm$ SE $1.9(n=4)$. To carry out the radioimmunoassays, a $10 \mu \mathrm{l}$ aliquot of each fecal extract was dried down and reconstituted in $100 \mu$ l working buffer $\left(0.1 \%\right.$ gelatin phosphate-buffered saline). ${ }^{125}$ I-labeled testosterone tracer $(50 \mu \mathrm{l})$ and $100 \mu \mathrm{l}$ antiserum (diluted 1:2) were added to sample or standards $(100 \mu 1)$. After vortexing and overnight incubation at room temperature, $500 \mu 1$ second antibody (diluted 1:2) was added, and the tubes were vortexed and incubated for $60 \mathrm{~min}$ at room temperature. Then the tubes were centrifuged at $2 g$ for $60 \mathrm{~min}$ at $4{ }^{\circ} \mathrm{C}$. The supernatant was aspirated and the radioactivity in the precipitant was counted on a gamma counter. Standard concentrations ranged from 1.5 to $25 \mathrm{pg} /$ tube. For the storage experiment samples, testosterone intra- and interassay coefficients of variation were 6.0 and $11.2 \%$ for a high control and 7.8 and $11.7 \%$ for a low control ( $n=3$ assays).

Physiological validation. Wasser et al. (2000) physiologically validated the ICN corticosterone antibody by demonstrating that it detects a rise in fGC concentrations in yellow baboons following an ACTH challenge. Stavisky et al. (1995) physiologically validated the Pantex progesterone antibody by demonstrating that it detects a rise in baboon $\mathrm{fP}$ during the luteal phase of the reproductive cycle, and that $\mathrm{fP}$ varies significantly with reproductive state in the yellow baboon. We assessed whether the ICN Total Estrogens antibody reflected gonadal function by comparing baboon fE concentrations in immature (2-4 years) and mature ( $>7$ years) non-pregnant females. We calculated the mean of two samples per individual for 10 individuals in each age category. The replicate samples were collected in the same month and year. Immature females had significantly lower fE concentrations than adult females $(t=-2.45, p=0.020)$. Similarly, we assessed whether the Equate Testosterone antibody reflected gonadal function by comparing baboon fT concentrations in immature (3-4 years, $n=10)$ and mature (8-9 years, $n=10)$ males. Samples were collected within two months of the same year. Immature males had significantly lower fT concentrations than adult males $(t=-2.30, p=0.034)$. Concentrations of all four steroids used in this study were also monitored across late pregnancy and lactation in yellow baboons (Lynch et al., submitted), which can be considered a further physiological validation of all antibodies used in this study.

\subsection{Data interpretation}

In addition to basic analyses of absolute differences as a function of storage condition, we asked three questions from the resultant endocrine data, as practical examples for assessing how robust results would be in each of the experimental conditions. First, we compared non-pregnant versus pregnant adult females in fE and $\mathrm{fP}$ concentrations, repeating the statistical analyses on values from each of the four conditions, to determine whether the conclusions were the same regardless of effects of storage. Second, we created four profiles of mean fGC concentration by baboon age class, each based on the same 29 fecal samples, but constructed separately from the four different storage conditions. Previous studies of baboon plasma suggest that glucocorticoids vary with age class (Sapolsky and Altmann, 1991). Here we determine if age-related patterns in fGC are retained regardless of storage condition of fecal samples. Finally, we ordered adult male fecal samples $(n=8)$ in terms of their fT concentrations, from highest to lowest, and compared this order across storage conditions.

\subsection{Statistical analyses}

Using the raw data of steroid concentration per $\mathrm{ng} / \mathrm{g}$ feces, mean percentages of initial values based on storage condition were calculated by dividing the mean concentration for each sample in conditions A, B or C by the mean concentration for the corresponding sample in condition I, and multiplying by 100 . For all other analyses, data were log transformed to meet assumptions of normality. Sample values in condition I were compared to those in $\mathrm{A}, \mathrm{B}$, and $\mathrm{C}$ using paired $t$ tests. Female baboon reproductive status is readily determined from external signals of cycling and pregnancy, and infants are typically seen within the first three days of birth, and so the reproductive condition was known for all females sampled. Fecal estrogen (fE) levels across reproductive states were compared using $t$ tests for samples with unequal variance, and fP levels were compared with $t$ tests for samples with equal variance, as fP samples from all conditions met the assumptions 
of Levene's test for equal variance. A Spearman rank test was used for all correlations. Significance levels for all tests were set at $p \leqslant 0.05$.

\section{Results}

\subsection{Condition A: two-week storage in $-20^{\circ} \mathrm{C}$ freezer}

Mean percentage of initial values for steroid concentrations of samples stored in condition A (see Table 1) are depicted in Fig. 1a. Under this storage condition, the mean percentage of initial values (condition I) for fGC was $92.5 \% \pm 2.3 \mathrm{SE}$, for $\mathrm{fE} 96.9 \% \pm 5.4 \mathrm{SE}$, for $\mathrm{fT}$ $115.4 \% \pm 9.1 \mathrm{SE}$, and for fP $107.7 \% \pm 6.0$ SE. Correlations between log-transformed sample concentrations in condition I and condition A were 0.918 for fGC, 0.973 for fE, 0.834 for $\mathrm{fT}$, and 0.881 for fP (all $p$ values $<0.001$ ). Condition A did not differ from condition I for $\mathrm{fE}$, fT, or $\mathrm{fP}$. However, fGC concentrations were

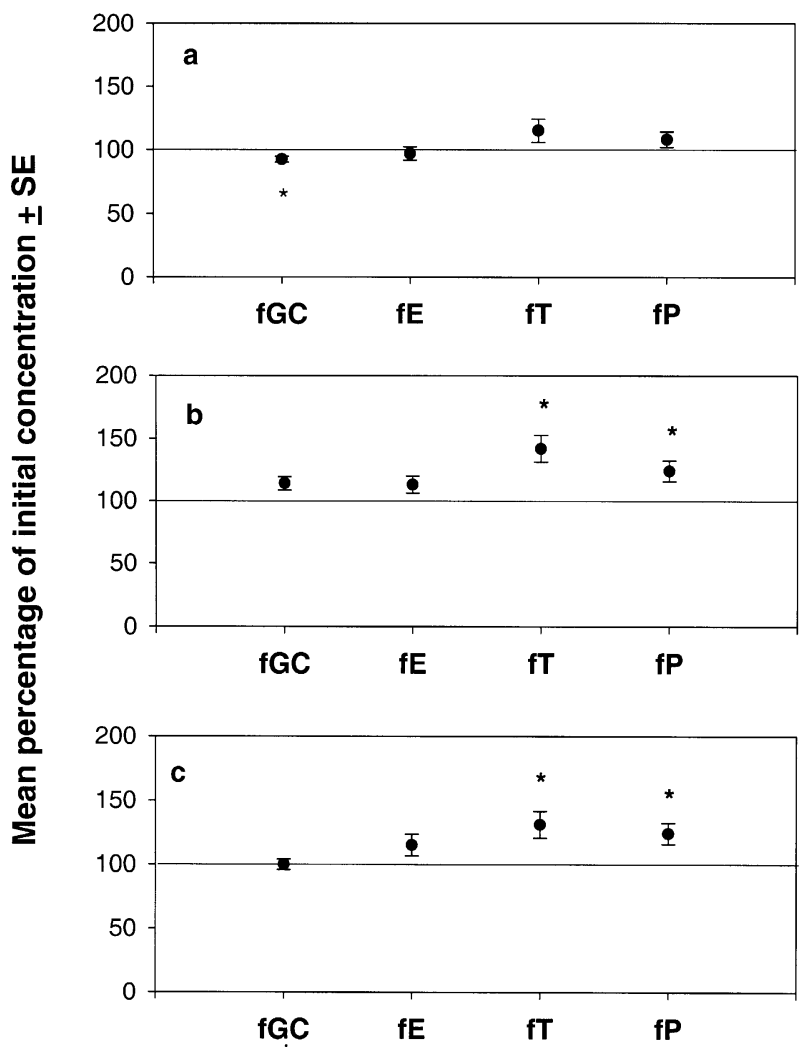

Fig. 1. Mean percentage of initial concentration $\pm \mathrm{SE}$ for fGC, fE, fT, and $\mathrm{fP}$ for fecal samples $(n=29)$ subjected to (a) two-week storage in a $-20^{\circ} \mathrm{C}$ freezer, (b) two-week field storage in a charcoal refrigerator, and (c) two-week field storage in a charcoal refrigerator followed by two-week storage in a $-20^{\circ} \mathrm{C}$ freezer, prior to lyophilization (see Table 1 and text for details). Initial concentrations (I) are calculated as the mean concentration when the samples were lyophilized two days after collection. Asterisks indicate a significant difference from the mean initial concentration at $p<0.05$. significantly lower in condition A than in condition I $(t=3.194, p=0.003)$.

\subsection{Condition B: two-week field storage in charcoal refrigerator}

Mean percentage of initial values for steroid concentrations of samples in condition B (see Table 1) are depicted in Fig. 1b. In condition B, the mean percentage of initial values (condition I) for fGC was $114.0 \% \pm 5.4 \mathrm{SE}$, for $\mathrm{fE} 113.3 \% \pm 6.8 \mathrm{SE}$, for fT $141.9 \% \pm 10.8 \mathrm{SE}$, and for fP $124.0 \% \pm 8.3 \mathrm{SE}$. Correlations between log-transformed sample concentrations in condition I and condition B were 0.726 for fGC, 0.962 for $\mathrm{fE}, 0.865$ for $\mathrm{fT}$, and 0.795 for $\mathrm{fP}$ (all $p$ values $<0.001$ ). Condition $\mathrm{B}$ did not differ from condition I in fE or fGC. However, both fT $(t=-3.854, p=0.001)$ and $\mathrm{fP}(t=-2.286, p=0.03)$ concentrations were significantly higher in condition $\mathrm{B}$ than in condition I.

3.3. Condition $C$ : two-week field storage in charcoal refrigerator followed by two-week storage in $-20^{\circ} \mathrm{C}$ freezer

Mean percentage of initial values for steroid concentrations of samples stored in condition C (see Table 1) are depicted in Fig. 1c. For these samples, the mean percentage of initial values (condition I) for fGC was $99.7 \% \pm 4.1 \mathrm{SE}$, for fE $115.1 \%+8.4 \mathrm{SE}$, for fT $130.6 \%$ $\pm 10.3 \mathrm{SE}$, and for fP $124.5 \% \pm 8.3 \mathrm{SE}$. Correlations between log-transformed sample concentrations in condition I and condition C were 0.791 for fGC, 0.949 for $\mathrm{fE}$, 0.811 for $\mathrm{fT}$, and 0.783 for fP (all $p$ values $<0.001$ ). Condition $\mathrm{C}$ did not differ from condition I for $\mathrm{fE}$ or fGC. However, both fT $(t=-2.096, p=0.045)$ and $\mathrm{fP}(t=-2.305, p=0.029)$ concentrations were significantly higher in condition $\mathrm{C}$ than to condition $\mathrm{I}$.

\subsection{Effect of storage condition on the interpretation of hormonal data}

Reproductive hormones and pregnancy. The comparison between pregnant and non-pregnant adult females in their $\mathrm{fE}$ and $\mathrm{fP}$ concentrations was robust across all four storage conditions (Fig. 2), with both fE and fP significantly higher in pregnant females ( $n=4$ samples) than in non-pregnant females ( $n=10$ samples), regardless of storage condition (fE: condition I, $t=-4.763, p=0.012$; $\mathrm{A}, t=-4.393, p=0.014 ; \mathrm{B}, t=-4.196, p=0.014 ; \mathrm{C}$, $t=-4.327, p=0.012 ; \quad \mathrm{fP}: \quad \mathrm{I}, t=-3.396, p=0.005$; $\mathrm{A}, t=3.729, p=0.003 ; \mathrm{B}, t=-3.269, p=0.007 ; \mathrm{C}$, $t=-4.592, p=0.001)$.

Glucocorticoids and age. Mean fGC concentration was calculated across age classes, using steroid concentration values from the four different storage conditions ( $n=29$ samples; Fig. 3). Comparing mean fGC of each 

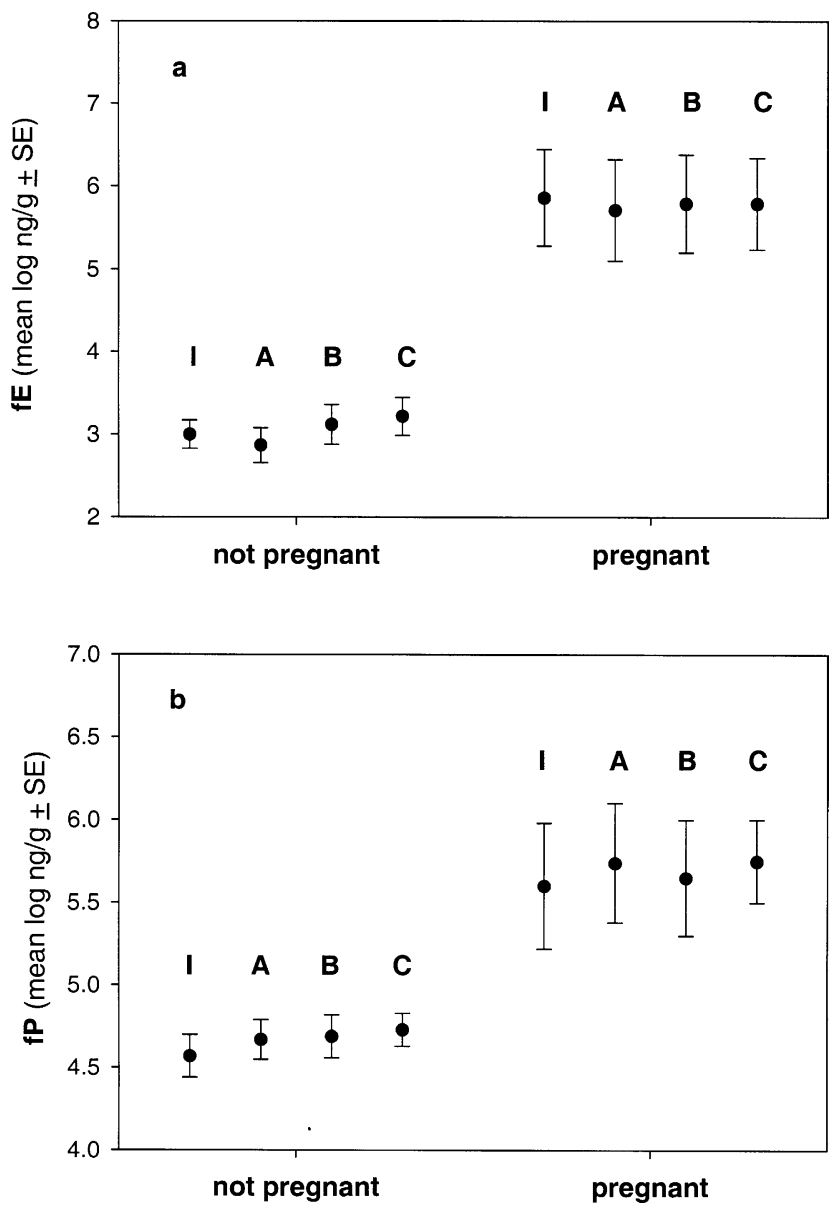

Fig. 2. Mean (a) fE and (b) fP ng/g feces \pm SE for pregnant $(n=4$ samples) and non- pregnant ( $n=10$ samples) adult females, based on the four experimental storage conditions (I, A, B, and C) of fecal samples (see text and Table 1 for description of each storage condition). Pregnant females exhibited significantly higher fE and fP concentrations than non-pregnant females, regardless of the conditions of storage of fecal samples.

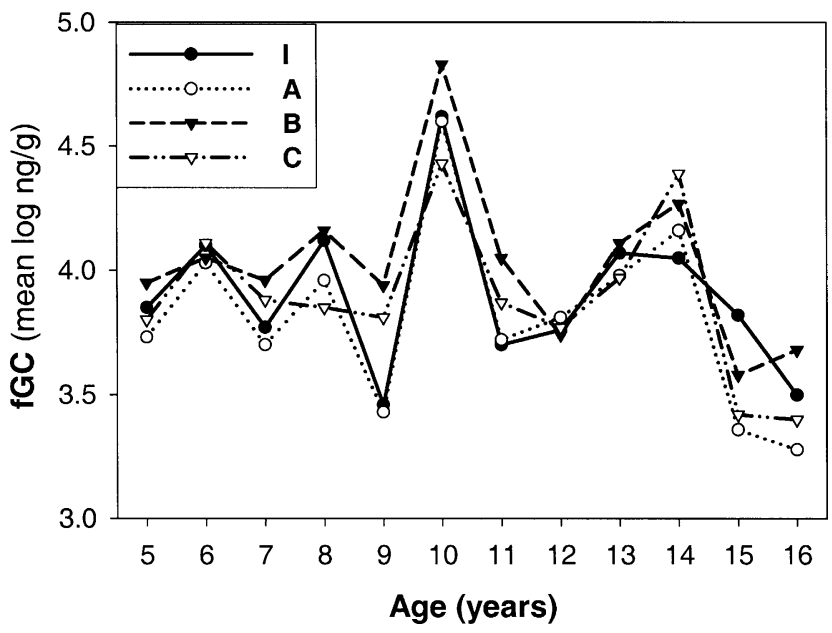

Fig. 3. Mean log fGC concentration by years of age, based on storage condition of fecal samples. The number of individual baboons sampled from each age category (5-16 years of age) ranged from 1 to 6 , for a total of 29 samples. Storage conditions I, A, B, and C are described in the text and Table 1.
Table 2

Testosterone values in adult males $(n=8)$, ordered from highest to lowest, by storage condition

\begin{tabular}{|c|c|c|c|c|}
\hline & I & A & $\mathrm{B}$ & $\mathrm{C}$ \\
\hline \multirow{3}{*}{ 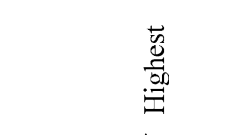 } & 1 & & 1 & 3 \\
\hline & & 1,2 & & \\
\hline & 2 & & \multirow[b]{2}{*}{3,5} & 5 \\
\hline \multirow{5}{*}{ 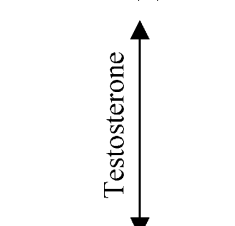 } & 3 & 3 & & 2 \\
\hline & 4 & 5 & 2 & \\
\hline & & & & 1,4 \\
\hline & & & 4 & \\
\hline & 5,6 & 4,6 & & \\
\hline+ & & & 6 & 6 \\
\hline 岕 & 7 & 7 & 7 & 7 \\
\hline (2) & 8 & 8 & 8 & 8 \\
\hline Minimum changes: & & 1 & 2 & 3 \\
\hline Step-wise changes: & & 1 & 3 & 6 \\
\hline
\end{tabular}

Initial testosterone concentrations (condition I) were ranked 1-8 in descending order. Those rank numbers, used to represent the eight samples, were then displayed in order from highest to lowest for conditions A, B, and C. Ties $( \pm 0.05 \mathrm{log} \mathrm{ng} / \mathrm{g})$ are displayed on the same line. We developed two indices to describe the distance of the treated samples from the initial order. For both indices, we broke ties in the direction of the original state. In the "minimum changes" index, we counted the number of samples that had moved out of the initial order, regardless of distance. In the "step-wise changes" index, we counted the minimum number of rank changes required to restore the treatment to the initial condition.

sampled age class ( $n=12)$ across storage conditions, the resultant data from each of the three experimental storage conditions and that from the initial condition were significantly correlated (Spearman's $r$ : condition A, $r=0.881, p=0.001 ; \quad$ condition $\mathrm{B}, \quad r=0.732, p=$ 0.007; condition $\mathrm{C}, r=0.643, p=0.024$ ).

Differences in male testosterone concentration. Fecal samples from adult males ( $n=8$ samples) were ordered from highest to lowest by fT concentration, and this rank ordering was compared by storage condition (Table 2). Condition A had only one reversal from condition I. In condition B, two samples moved out of the original order. Condition $\mathrm{C}$ had the highest degree of change from the initial order, using either the "minimum changes" or the "step-wise changes" index (see Table 2).

\section{Discussion}

As expected, the best alternative to prompt lyophilization was to freeze fecal samples at $-20^{\circ} \mathrm{C}$ for two weeks prior to lyophilization (condition A). This storage method resulted in no significant change from initial steroid concentrations for $\mathrm{fE}, \mathrm{fT}$, or $\mathrm{fP}$. As found in a previous experiment after 30 days of subzero storage (Khan et al., 2002), fGC significantly decreased in mean concentration after two weeks of frozen storage 
compared to initial values. However, this decrease was slight (frozen samples were $92 \% \pm 2.3 \mathrm{SE}$ of initial concentrations), and significance was due in large part to the consistency of the decline across the 29 samples.

Storage for two weeks in the charcoal refrigerator (condition B) caused a mean increase in all four steroid concentrations, which reached significance for $\mathrm{fT}$ $(141.9 \% \pm 10.8 \mathrm{SE})$, and for fP $(124.0 \% \pm 8.3 \mathrm{SE})$. However, this storage condition was resilient in terms of practical questions asked of the data. Fecal E and fP values still reflected pregnant versus non-pregnant states in baboon females, a fGC profile constructed by age class was qualitatively similar to that created from the immediately lyophilized samples, although slightly inflated across age classes, and there were only moderate changes in relative fT concentrations across adult males.

The effects of storage for two weeks in the charcoal refrigerator followed by two weeks in the $-20^{\circ} \mathrm{C}$ freezer (condition $\mathrm{C}$ ) appeared superficially similar to charcoal refrigerator storage alone. However, mean fGC and fT declined in steroid concentration compared to charcoal storage only. It appears that for fGC, and perhaps for $\mathrm{fT}$, concentrations tend to increase in ambient temperature storage, but may decline, at least initially (Khan et al., 2002), when subjected to sub-zero storage. The use of freezing after ambient storage seemed to ameliorate, on average, the effects of increasing values. However, this mean advantage was achieved at the cost of greater variability in the direction of steroid concentration change across individual samples, so that relative patterns in steroid concentration were sometimes obscured (see Table 2 for fT and Fig. 3 for fGC).

If samples from different storage conditions or storage durations are analyzed indiscriminately together for hormone concentration, scatter increases and may dampen the ability to see real biological differences between groups or individuals. As seen in Fig. 3, if samples are taken at random from each storage condition, some of which tend to decrease sample values and others which tend to inflate values, the margin of error increases with each addition of a different storage protocol. Increased sample size, and repeated sampling of individuals, may be enough to elucidate real patterns when the differences in storage conditions among fecal samples analyzed are small. However, extrapolating from the results of Khan and colleagues' (2002) mediumterm storage experiment, it is easy to imagine that fecal samples collected over a year and stored together at ambient temperature until the end of that year, might upon subsequent analysis present a hormonal profile that mimicked seasonal variation in steroid concentration, but was in fact entirely an artifact of the differential durations of sample storage. Consequently, not only validation for the storage conditions, but limitations on the range of storage duration, may be essential for valid results when using fecal steroid analysis in the field.
The results presented here point to the importance of validation of storage methods for each hormone, as "ideal" storage conditions may not be the same for all steroids. For example, short-term freezing of samples maintained stability in $\mathrm{fP}$ concentration, but led to a significant decline in fGC; in contrast, a combination of 'ambient' temperature storage followed by freezing led to a significant increase in $\mathrm{fP}$ but no change in fGC. Knowledge of the effects of storage, coupled with the scope of questions to be asked, the specific steroids to be analyzed in one's study, and practical logistics influencing feasibility of storage methods, will together help to determine the optimal compromise for storage protocol in a particular research project. The present study provides both concrete results for four steroids in baboons and a model for a comparative approach of storage evaluation applicable to diverse mammalian species.

\section{Acknowledgments}

Supported by the Chicago Zoological Society, NSF IBN-9983910 and its predecessors, and 1 R03 MH65294. Appreciation is due to the Office of the President, Republic of Kenya, the Kenya Wildlife Services, its Amboseli staff and Wardens, the Institute of Primate Research and especially J.M. Mwenda, S.F. Dossaji of the National Museums of Kenya, and the members of the Amboseli-Longido pastoralist communities. Particular thanks go to Amboseli Baboon Research Project co-director S.C. Alberts and to the Amboseli fieldworkers who contributed to sample and data collection-especially J.K. Warutere, R. Mututua, and S. Sayialel. P. Whitten and $\mathrm{T}$. Ziegler provided valuable comments and advice on assay validation and fecal steroid analysis as we established techniques in our laboratory. Comments from S.C. Alberts, T.C. Good, R.E. Pride, M.E. Alfaro, and two anonymous reviewers greatly improved the manuscript.

\section{References}

Boinski, S., Swing, S.P., Gross, T.S., Davis, J.K., 1999. Environmental enrichment of brown capuchins (Cebus apella): behavioral and plasma and fecal cortisol measures of effectiveness. American Journal of Primatology 48, 49-68.

Brockman, D.K., Whitten, P.L., Richard, A.F., Benander, B., 2001. Birth season testosterone levels in male Verreaux's sifaka, Propithecus verreauxi: insights into socio-demographic factors mediating seasonal testicular function. Behavioral Ecology and Sociobiology $49,117-127$

Brockman, D.K., Whitten, P.L., Richard, A.F., Schneider, A., 1998. Reproduction in free-ranging male Propithecus verreauxi: the hormonal correlates of mating and aggression. American Journal of Physical Anthropology 105, 137-151.

Brown, J.L., Wemmer, C.M., Lehnardt, J., 1995. Urinary cortisol analysis for monitoring adrenal activity in elephants. Zoo Biology $14,533-542$. 
Campbell, C.J., Shideler, S.E., Todd, H.E., Lasley, B.L., 2001. Fecal analysis of ovarian cycles in female black-handed spider monkeys (Ateles geoffroyi). American Journal of Primatology 54, 79-89.

Cavigelli, S., 1999. Behavioural patterns associated with faecal cortisol levels in free-ranging female ring-tailed lemurs, Lemur catta. Animal Behaviour 57, 935-944.

Creel, S., Creel, N.M., Monfort, S.L., 1996. Social stress and dominance. Nature 379, 212.

Dehnhard, M., Clauss, M., Lechner-Doll, M., Meyer, H.H.D., Palme, R., 2001. Noninvasive monitoring of adrenocortical activity in roe deer (Capreolus capreolus) by measurement of fecal cortisol metabolites. General and Comparative Endocrinology 123, 111-120.

Garnier, J.N., Green, D.L, Pickard, A.R., Shaw, H.I., Holt, W.V., 1998. Non-invasive diagnosis of pregnancy in wild black rhinoceros (Diceros bicornis minor) by faecal steroid analysis. Reproduction, Fertility and Development 10, 451-458.

Goymann, W., Moestl, E., Van’t Hof, T., East, M.L., Hofer, H., 1999. Noninvasive fecal monitoring of glucocorticoids in spotted hyenas, Crocuta crocuta. General and Comparative Endocrinology 114, 340-348.

Grant, J.K., Beastall, G.H., 1983. Clinical Biochemistry of Steroid Hormones: Methods and Applications. Elsevier, New York.

Heistermann, M., Möhle, U., Vervaecke, H., van Elsacker, L., Hodges, J.K., 1996. Application of urinary and fecal steroid measurements for monitoring ovarian function and pregnancy in the bonobo (Pan paniscus) and evaluation of perineal swelling patterns in relation to endocrine events. Biology of Reproduction 55, 844-853.

Heistermann, M., Uhrigshardt, J., Husung, A., Kaumanns, W., Hodges, J.K., 2001. Measurement of faecal steroid metabolites in the lion-tailed macaque (Macaca silenus): a non-invasive tool for assessing female ovarian function. Primate Report 59, 27-42.

Jurke, M.H., Czekala, N.M., Fitch-Snyder, H., 1997. Non-invasive detection and monitoring of estrus, pregnancy and the postpartum period in pygmy loris (Nycticebus pygmaeus) using fecal estrogen metabolites. American Journal of Primatology 41, 103-115.

Kesner, J.S., Knecht, E.A., Krieg Jr., E.F., 1995. Stability of urinary female reproductive hormones stored under various conditions. Reproductive Toxicology 9, 239-244.

Khan, M.Z., Altmann, J., Isani, S.S., Yu, J., 2002. A matter of time: evaluating the storage of fecal samples for steroid analysis. General and Comparative Endocrinology 128, 57-64.

Kraus, C., Heistermann, M., Kappeler, P.M., 1999. Physiological suppression of sexual function of subordinate males: a subtle form of intrasexual competition among male sifakas (Propithecus verreauxi)? Physiology and Behavior 66, 855-861.

Lasley, B.L., Kirkpatrick, J.F., 1991. Monitoring ovarian function in captive and free-ranging wildlife by means of urinary and fecal steroids. Journal of Zoo and Wildlife Medicine 22, 23-31.

Lynch, J.W., Ziegler, T.E., Strier, K.B., 2002. Individual and seasonal variation in fecal testosterone and cortisol levels of wild tufted capuchin monkeys, Cebus apella nigritus. Hormones and Behavior 41, 275-287.

Lynch, J.W., Nguyen, N., Altmann, J., Alberts, S.C. (submitted). Steroid concentrations in wild peri-parturitional baboons, Papio cynocephalus: interactions with life history variables.

Möstl, E., Messmann, S., Bagu, E., Robia, C., Palme, R., 1999. Measurement of glucocorticoid metabolite concentrations in faeces of domestic livestock. Journal of Veterinary Medicine A 46, 621-631.

Ostner, J., Kappeler, P.M., Heistermann, M., 2002. Seasonal variation and social correlates of androgen excretion in male redfronted lemurs (Eulemur fulvus rufus). Behavioral Ecology and Sociobiology 52, 485-495.

Sapolsky, R.M., Altmann, J., 1991. Incidence of hypercortisolism and dexamethasone resistance increases with age among wild baboons. Biological Psychiatry 30, 1008-1016.
Schwarzenberger, F., Palme, R., Bamberg, E., Möstl, E., 1997. A review of faecal progesterone metabolite analysis for non-invasive monitoring of reproductive function in mammals. Journal of Mammalian Biology 62, 214-221.

Shideler, S.E., Savage, A., Ortuño, A.M., Moorman, E.A., Lasley, B.L., 1994. Monitoring female reproductive function by measurement of fecal estrogen and progesterone metabolites in the whitefaced saki (Pithecia pithecia). American Journal of Primatology 32, 95-108.

Stavisky, R., Russell, E., Stallings, J., Smith, E.O., Worthman, C., Whitten, P.L., 1995. Fecal steroid analysis of ovarian cycles in free-ranging baboons. American Journal of Primatology 36, 285297.

Strier, K.B., Ziegler, T.E., 1994. Insights into ovarian function in wild muriqui monkeys (Brachyteles arachnoides). American Journal of Primatology 32, 31-40.

Strier, K.B., Ziegler, T.E., 2000. Lack of pubertal influences on female dispersal in muriqui monkeys, Brachyteles arachnoides. Animal Behaviour 59, 849-860.

Strier, K.B., Ziegler, T.E., Wittwer, D., 1999. Seasonal and social correlates of fecal testosterone and cortisol levels in wild male muriquis (Brachyteles arachnoides). Hormones and Behavior 35, 125-134.

Terio, K.A., Brown, J.L., Moreland, R., Munson, L., 2002. Comparison of different drying and storage methods on quantifiable concentrations of fecal steroids in the cheetah. Zoo Biology 21, 119-134.

Washburn, B.E., Millspaugh, J.J., 2002. Effects of simulated environmental conditions on glucocorticoid metabolite measurements in white-tailed deer feces. General and Comparative Endocrinology 127, 217-222.

Wasser, S.K., Belloso, A.D., Rodden, N.M., 1995. Using fecal steroids to evaluate reproductive function in female maned wolves. Journal of Wildlife Management 59, 889-894.

Wasser, S.K., Hunt, K.E., Brown, J.L., Cooper, K., Crockett, C.M., Bechert, U., Millspaugh, J.J., Larson, S., Monfort, S.L., 2000. A generalized fecal glucocorticoid assay for use in a diverse array of nondomestic mammalian and avian species. General and Comparative Endocrinology 120, 260-275.

Wasser, S.K., Papageaorge, S., Foley, C., Brown, J.L., 1996. Excretory fate of estradiol and progesterone in the African elephant (Loxodonta africana) and patterns of fecal steroid concentrations throughout the estrous cycle. General and Comparative Endocrinology 102, 255-262.

Wasser, S.K., Risler, L., Steiner, R.A., 1988. Excreted steroids in primate feces over the menstrual cycle and pregnancy. Biology of Reproduction 39, 862-872.

Wasser, S.K., Thomas, R., Nair, P.P., Guidry, C., Southers, J., Lucas, J., Wildt, D.E., Monfort, S.L., 1993. Effects of dietary fiber on fecal steroid measurements in baboons (Papio cynocephalus cynocephalus). Journal of Reproduction and Fertility 97, 569-574.

Whitten, P.L., Brockman, D.K., Stavisky, R.C., 1998. Recent advances in noninvasive techniques to monitor hormone-behavior interactions. Yearbook of Physical Anthropology 41, 1-23.

Ziegler, T., Hodges, K., Winkler, P., Heistermann, M., 2000. Hormonal correlates of reproductive seasonality in wild female Hanuman langurs (Presbytis entellus). American Journal of Primatology 51, 119-134.

Ziegler, T.E., Scheffler, G., Wittwer, D.J., Schultz-Darken, N.J., Snowdon, C.T., Abbott, D.H., 1996. Metabolism of reproductive steroids during the ovarian cycle in two species of callitrichids, Saguinus oedipus and Callithrix jacchus, and estimation of the ovulatory period from fecal steroids. Biology of Reproduction 54, 91-99. 